\title{
A VARIATIONAL MCSHANE CHARACTERIZATION OF LOCALLY CONVEX SPACES POSSESSING THE RADON-NIKODYM PROPERTY
}

\author{
Sokol Bush KaliaJ
}

\begin{abstract}
We present a characterization of complete locally convex topological vector spaces possessing the Radon-Nikodym property in terms of additive interval functions whose McShane variational measures are absolutely continuous with respect to the Lebesgue measure.
\end{abstract}

\section{Introduction and Preliminaries}

In the papers [2] and [5], characterizations of Banach spaces possessing the Radon-Nikodym property are given in terms of additive interval functions whose Henstock and McShane variational measures are absolutely continuous with respect to the Lebesgue measure. There are also characterizations of the Radon-Nikodym property in terms of the limit average range and the average range of additive interval functions, see [6], [7] and [8. In the paper [5], it is shown that a Banach space $X$ has the Radon-Nikodym property if and only if for every $X$-valued additive interval function $\varphi$. which has absolutely continuous McShane variational measure there exists a McShane integrable function $f:[0,1] \rightarrow X$ such that

$$
\varphi(I)=(M) \int_{I} f \quad \text { for all closed subinterval } \quad I \subset[0,1]
$$

and $f$ is weakly equivalent to a measurable function $g$. Here, we extend this result to a complete locally convex topological vector space $X$, Theorem 2.3. Due to this result, several $X$-valued set functions that are only additive can be represented as McShane integrals.

(C) 2014 Mathematical Institute, Slovak Academy of Sciences.

2010 Mathematics Subject Classification: Primary 28B05, 46B22, 26A46, 46A03; Secondary 46G10, 46G05.

Keywords: Radon-Nikodym property, complete locally convex topological vector spaces, additive interval function, McShane variational measure. 
Let $X$ be a Hausdorff locally convex topological vector space (briefly a locally convex space) with topological dual $X^{\prime}$. By $\mathscr{P}$ the family of all continuous seminorms in $X$ is denoted. For any $p \in \mathscr{P}$, we denote by $\widetilde{X}_{p}$ the quotient vector space $X / p^{-1}(0)$, by $\phi_{p}: X \rightarrow \widetilde{X}_{p}$ the canonical quotient map, by $\left(\widetilde{X}_{p}, \widetilde{p}\right)$ the quotient normed space and by $\left(\bar{X}_{p}, \bar{p}\right)$ the completion of $\left(\widetilde{X}_{p}, \widetilde{p}\right)$. Note that

$$
X^{\prime}=\left\{\widetilde{v}_{p}^{\prime} \circ \phi_{p}: p \in \mathscr{P}, \widetilde{v}_{p}^{\prime} \in \widetilde{X}_{p}^{\prime}\right\}
$$

where $\widetilde{X}_{p}^{\prime}$ is the topological dual of $\left(\widetilde{X}_{p}, \widetilde{p}\right)$. For every $p, q \in \mathscr{P}$, such that $p \leq q$, we denote by $\widetilde{g}_{p q}: \widetilde{X}_{q} \rightarrow \widetilde{X}_{p}$ the map defined as follows

$$
\widetilde{g}_{p q}\left(w_{q}\right)=w_{p} \quad \text { for each } \quad w_{q} \in \tilde{X}_{q}
$$

where $w_{p}=\phi_{p}(x)$, for some vector $x \in w_{q}$. By $\bar{g}_{p q}$ the continuous linear extension of $\widetilde{g}_{p q}$ to $\bar{X}_{q}$ is denoted.

We denote by $\mathcal{I}$ the family of all non-degenerate closed subintervals of $[0,1]$, by $\lambda$ the Lebesgue measure and by $\mathcal{L}$ the family of all Lebesgue measurable subsets of $[0,1]$. The two intervals $I, J \in \mathcal{I}$ are said to be nonoverlapping if $\operatorname{int}(I) \cap \operatorname{int}(J)=\emptyset$, where $\operatorname{int}(I)$ denotes the interior of $I$. If a point function $F:[0,1] \rightarrow X$ is given, then we denote by $\widetilde{F}$ the interval function $\widetilde{F}: \mathcal{I} \rightarrow X$ defined by $\widetilde{F}([u, v])=F(v)-F(u)$, for all $[u, v] \in \mathcal{I}$. An interval function $\varphi: \mathcal{I} \rightarrow X$ is said to be additive if for each two nonoverlapping intervals $I, J \in \mathcal{I}$ with $I \cup J \in \mathcal{I}$, we have

$$
\varphi(I \cup J)=\varphi(I)+\varphi(J) .
$$

A function $F:[0,1] \rightarrow X$ is said to be differentiable by seminorms if there is a function $f:[0,1] \rightarrow X$ satisfying the following property: given $p \in \mathscr{P}$, there exists a subset $Z_{p} \subset[0,1]$ with $\lambda\left(Z_{p}\right)=0$, such that

$$
\lim _{h \rightarrow 0} p\left(\frac{F(t+h)-F(t)}{h}-f(t)\right)=0 \quad \text { for all } t \in[0,1] \backslash Z_{p} .
$$

A pair $(I, t)$ of an interval $I \in \mathcal{I}$ and a point $t \in[0,1]$ is said to be a McShane tagged interval, $t$ is said to be the tag of $I$. A Mcshane partition (M-partition) in $[0,1]$ is a finite collection of McShane tagged intervals $(I, t)$ whose corresponding intervals are nonoverlapping. A function $\delta: E \rightarrow(0,+\infty)$ is said to be a gauge on $E \subset[0,1]$. We say that an $\mathcal{M}$-partition $\pi$ in $[0,1]$ is:

- an $\mathcal{M}$-partition of $[0,1]$ if $\bigcup_{(I, t) \in \pi} I=[0,1]$,

- E-tagged if for each $(I, t) \in \pi$, we have $t \in E$,

- $\delta$-fine, if for every $(I, t) \in \pi$, we have $I \subset(t-\delta(t), t+\delta(t))$.

Let us now recall the definitions of McShane and variational McShane integrals. We refer to [9], 10] and [11] for more information about McShane and variational McShane integrals of functions taking values in a locally convex space. 


\section{MCSHANE INTEGRAL AND THE RADON-NIKODYM PROPERTY}

Definition 1.1. A function $f:[0,1] \rightarrow X$ is said to be McShane integrable on $[0,1]$, if there exists a vector $w \in X$ satisfying the following property: given $\varepsilon>0$ and $p \in \mathscr{P}$, there exists a gauge $\delta_{p}^{\varepsilon}$ on $[0,1]$ such that for every $\delta_{p}^{\varepsilon}$-fine, $\mathcal{M}$-partition $\pi$ of $[0,1]$, we have

$$
p\left(\sum_{(I, t) \in \pi} f(t) \lambda(I)-w\right)<\varepsilon .
$$

We denote $(M) \int_{[0,1]} f=w$. A function $f:[0,1] \rightarrow X$ is said to be McShane integrable on $E \subset[0,1]$ if the function $f \cdot \chi_{E}:[0,1] \rightarrow X$ is McShane integrable on $[0,1]$, and we denote

$$
(M) \int_{[0,1]} f \cdot \chi_{E}=(M) \int_{E} f,
$$

where $\chi_{E}$ is the characteristic function of $E$.

Definition 1.2. We say that a function $f:[0,1] \rightarrow X$ is variational McShane integrable on $[0,1]$, if there is a function $F:[0,1] \rightarrow X$ satisfying the following property: given $\varepsilon>0$ and $p \in \mathscr{P}$, there exists a gauge $\delta_{p}^{\varepsilon}$ on $[0,1]$ such that for every $\delta_{p}^{\varepsilon}$-fine, $\mathcal{M}$-partition $\pi$ of $[0,1]$, we have

$$
\sum_{(I, t) \in \pi} p(f(t) \lambda(I)-\widetilde{F}(I))<\varepsilon .
$$

We say that $F$ is the primitive of $f$. If $f$ is variational McShane integrable with the primitive $F$, then $f$ is McShane integrable and

$$
\widetilde{F}(I)=(M) \int_{I} f \quad \text { for all } \quad I \in \mathcal{I} \text {. }
$$

Given $F:[0,1] \rightarrow X, p \in \mathscr{P}$, a subset $E \subset[0,1]$ and gauge $\delta$ on $E$, we define

$$
V_{(p, F)}^{\mathcal{M}}(E, \delta)=\sup \sum_{(I, t) \in \pi} p(\widetilde{F}(I)),
$$

where supremum is taken over all $E$-tagged, $\delta$-fine, $\mathcal{M}$-partition $\pi$ in $[0,1]$. Then, we set

$$
V_{(p, F)}^{\mathcal{M}}(E)=\inf \left\{V_{(p, F)}^{\mathcal{M}}(E, \delta): \delta \text { is a gauge on } E\right\} .
$$

The set function $V_{p, F}^{\mathcal{M}}($.$) is said to be the McShane variational measure generated$ by $F$ with respect to seminorm $p$. By B. Thom s o n's results from [15] or [16], it is known that the set function $V_{p, F}^{\mathcal{M}}($.$) is a Borel metric outer measure on [0,1]$.

We denote by $\mathcal{V}_{(p)}^{\mathcal{M}}$ the class of all functions $F:[0,1] \rightarrow X$ such that for each $E \in \mathcal{L}$, we have

$$
\lambda(E)=0 \Rightarrow V_{(p, F)}^{\mathcal{M}}(E)=0 .
$$


The function $F:[0,1] \rightarrow X$ is said to be strongly absolutely continuous $(s A C)$ if, for each $p \in \mathscr{P}$, the function $\phi_{p} \circ F$ is $s A C$.

A function $f:[0,1] \rightarrow X$ is said to be measurable by seminorms if, for each $p \in \mathscr{P}$, there exists a sequence $\left(f_{k}^{(p)}\right)_{k}$ of measurable simple functions and a subset $Z_{p} \subset[0,1]$ with $\lambda\left(Z_{p}\right)=0$, such that

$$
\lim _{k \rightarrow \infty} p\left(f_{k}^{(p)}(t)-f(t)\right)=0 \quad \text { for all } t \in[0,1] \backslash Z_{p} .
$$

The function $f:[0,1] \rightarrow X$ is said to be weakly measurable, if $x^{\prime} f$ is measurable for all $x^{\prime} \in X^{\prime}$.

Two weakly measurable functions $f:[0,1] \rightarrow X$ and $g:[0,1] \rightarrow X$ are said to be weakly equivalent if, for every $x^{\prime} \in X^{\prime}$, there is a subset $Z^{\left(x^{\prime}\right)} \subset[0,1]$ with $\lambda\left(Z^{\left(x^{\prime}\right)}\right)=0$ such that

$$
x^{\prime} f(t)=x^{\prime} g(t) \quad \text { for all } \quad t \in[0,1] \backslash Z^{\left(x^{\prime}\right)} .
$$

Definition 1.3. A function $f:[0,1] \rightarrow X$ is said to be integrable by seminorms if, for any $p \in \mathscr{P}$, there exists a sequence $\left(f_{k}^{(p)}\right)_{k}$ of measurable simple functions and a subset $Z_{p} \subset[0,1]$ with $\lambda\left(Z_{p}\right)=0$, such that:

(i) for all $t \in[0,1] \backslash Z_{p}$, we have

$$
\lim _{k \rightarrow \infty} p\left(f_{k}^{(p)}(t)-f(t)\right)=0
$$

(ii) each function $p \circ\left(f_{k}^{(p)}-f\right)$ is Lebesgue integrable on $[0,1]$ and

$$
\lim _{k \rightarrow \infty} \int_{[0,1]} p\left(f_{k}^{(p)}(t)-f(t)\right) d \lambda=0,
$$

(iii) for each $E \in \mathcal{L}$, there exists a vector $v_{E} \in V$ such that

$$
\lim _{k \rightarrow \infty} p\left(\int_{E} f_{k}^{(p)}(t) d \lambda-v_{E}\right)=0 .
$$

In this case, we put $\int_{E} f d \lambda=v_{E}$.

We refer to [1] for more information about the integrability by seminorms. This notion coincides with Bochner integrability in a Banach space.

A countable additive vector measure $\nu: \mathcal{L} \rightarrow X$ is said to be of bounded variation if, for each $p \in \mathscr{P}$, we have that the countable additive vector measure $\phi_{p} \circ \nu$ is of bounded variation. The countable additive vector measure $\nu$ is said to be $\lambda$-continuous if, for each $E \in \mathcal{L}$, we have

$$
\lambda(E)=0 \Rightarrow \nu(E)=0
$$

It is easy to see that $\nu$ is $\lambda$-continuous, if and only if each $\phi_{p} \circ \nu$ is $\lambda$-continuous. 


\section{MCSHANE INTEGRAL AND THE RADON-NIKODYM PROPERTY}

We say that $X$ has the Radon-Nikodym property (the RNP) if, for each countable additive $\lambda$-continuous vector measure $\nu: \mathcal{L} \rightarrow X$ of bounded variation, there is an integrable by seminorms function $f:[0,1] \rightarrow X$ such that

$$
\nu(E)=\int_{E} f d \lambda \quad \text { for all } \quad E \in \mathcal{L} .
$$

\section{The main result}

The main result is Theorem 2.3. Let us start with two auxiliary lemmas. We refer to [3] for the notions used in the following lemma.

Lemma 2.1. Let $(X,\|\|$.$) be a Banach space and let F:[0,1] \rightarrow X$ be a function. If $F$ is $s A C$, then there exists a unique countably additive $\lambda$-continuous vector measure $F_{\mathcal{L}}: \mathcal{L} \rightarrow X$ such that

$$
\widetilde{F}(I)=F_{\mathcal{L}}(I) \quad \text { for all } \quad I \in \mathcal{I} .
$$

Moreover, $F_{\mathcal{L}}$ is of bounded variation.

P r o o f. Let $\mathcal{I}_{0}$ be the set of all subintervals $I \subset[0,1]$ having one of two forms $[0, b]$ or $(a, b]$, where $0<a<b \leq 1$. For such intervals, place

$$
\widetilde{F}_{0}([0, b])=\widetilde{F}([0, b]) \text { and } \quad \widetilde{F}_{0}((a, b])=\widetilde{F}([a, b]) .
$$

Let $\mathscr{A}$ consist of all finite unions of such intervals. It is clear that $\mathscr{A}$ is an algebra and that if a set $E \in \mathscr{A}$ has the form

$$
E=I_{1} \cup I_{2} \cup \cdots \cup I_{n},
$$

where $I_{1}, I_{2}, \ldots, I_{n}$ are pairwise disjoint intervals of type described, then

$$
\widetilde{F}_{0}\left(I_{1}\right)+\widetilde{F}_{0}\left(I_{2}\right)+\cdots+\widetilde{F}_{0}\left(I_{n}\right)
$$

is independent of the particular family of pairwise disjoint intervals $I_{1}, I_{2}, \ldots, I_{n}$, whose union is $E$. Thus, we may define the vector $F_{\mathscr{A}}(E)$ by the equation

$$
F_{\mathscr{A}}(E)=\widetilde{F}_{0}\left(I_{1}\right)+\widetilde{F}_{0}\left(I_{2}\right)+\cdots+\widetilde{F}_{0}\left(I_{n}\right) .
$$

By Definition I.1.1 in [3], the set function $F_{\mathscr{A}}: \mathscr{A} \rightarrow X$ is a vector measure. Clearly, we have that $F_{\mathscr{A}}: \mathscr{A} \rightarrow X$ is a unique vector measure such that

$$
F_{\mathscr{A}}(I)=\widetilde{F}_{0}(I)=\widetilde{F}(\bar{I}) \quad \text { for all } \quad I \in \mathcal{I}_{0},
$$

where $\bar{I}$ is the closure of $I$. Since $F$ is $s A C$, we get that $F_{\mathscr{A}}$ is of bounded variation and

$$
\lim _{\substack{\lambda(A) \rightarrow 0 \\(A \in \mathscr{A})}} F_{\mathscr{A}}(A)=0 .
$$




\section{SOKOL BUSH KALIAJ}

Let us consider a sequence $\left(A_{n}\right)$ of pairwise disjoint members of $\mathscr{A}$ such that $A=\cup_{n=1}^{\infty} A_{n} \in \mathscr{A}$. Since

$$
\lim _{n \rightarrow \infty} \lambda\left(A \backslash \cup_{i=1}^{n} A_{i}\right)=0
$$

we obtain by (2.3) that

$$
\lim _{n \rightarrow \infty}\left\|F_{\mathscr{A}}(A)-\cup_{i=1}^{n} F_{\mathscr{A}}\left(A_{i}\right)\right\|=\lim _{n \rightarrow \infty}\left\|F_{\mathscr{A}}\left(A \backslash \cup_{i=1}^{n} A_{i}\right)\right\|=0 .
$$

Thus, $F_{\mathscr{A}}$ is a countable additive measure on $\mathcal{A}$, and since it is of bounded variation, we obtain in [3, Proposition I.1.15] that $F_{\mathscr{A}}$ is also strongly additive. Consequently, by Carathéodory-Hahn-Kluvánek Extension Theorem in [3], $F_{\mathscr{A}}$ has a unique countable additive extension $F_{\mathscr{B}}: \mathscr{B} \rightarrow X$, where $\mathscr{B}$ is $\sigma$-algebra generated by $\mathscr{A}$.

Claim 1. The vector measure $F_{\mathscr{B}}$ is $\lambda$-continuous. To see this, let us consider the semimetric space $\mathscr{B}(\lambda)$ consisting of members of $\mathscr{B}$ equipped with the semimetric

$$
\rho\left(B, B^{\prime}\right)=\lambda\left(B \Delta B^{\prime}\right),
$$

where $B \Delta B^{\prime}=\left(B \backslash B^{\prime}\right) \cup\left(B^{\prime} \backslash B\right)$. In [4, Lemma III.7.1], $\mathscr{A}(\lambda)$ consisting of elements of $\mathscr{A}$ is dense in $\mathscr{B}(\lambda)$. By (2.3) and

$$
F_{\mathscr{A}}(A)-F_{\mathscr{A}}\left(A^{\prime}\right)=F_{\mathscr{A}}\left(A \backslash A \cap A^{\prime}\right)-F_{\mathscr{A}}\left(A^{\prime} \backslash A \cap A^{\prime}\right),
$$

the function $F_{\mathscr{A}}: \mathscr{A} \rightarrow X$ is uniformly continuous. Consequently, $F_{\mathscr{B}}$ is the unique uniformly continuous extension of $F_{\mathscr{A}}$. Hence,

$$
\lim _{\substack{(B \Delta \emptyset) \rightarrow 0 \\(B \in \mathscr{B})}}\left\|F_{\mathscr{B}}(B)\right\|=0 .
$$

Therefore, by [3, Theorem I.2.1], it follows that $F_{\mathscr{B}}$ is $\lambda$-continuous.

Claim 2. The vector measure $F_{\mathscr{B}}$ is of bounded variation. To see this, we consider finite collection $\left\{B_{i}: i=1,2, \ldots, m\right\}$ of pairwise disjoint members of $\mathscr{B}$ and let $0<\varepsilon<1$ be given. Then, there exists $\delta>0$ such that for each $B, B^{\prime} \in \mathscr{B}$, we have

$$
\lambda\left(B \Delta B^{\prime}\right)<\delta \Rightarrow\left\|F_{\mathscr{B}}(B)-F_{\mathscr{B}}\left(B^{\prime}\right)\right\|<\frac{\varepsilon}{2 \cdot m^{2}} .
$$

Since $\mathscr{A}$ is dense in $\mathscr{B}(\lambda)$, for each $B_{i}$ there is an $A_{i}$ such that

Note that

$$
\lambda\left(B_{i} \Delta A_{i}\right)<\frac{\delta}{2}
$$

$$
\lambda\left(A_{i} \cap A_{j} \Delta \emptyset\right)<\delta \quad(i \neq j)
$$

because

$$
\left(A_{i} \cap A_{j}\right) \backslash B_{i} \subset A_{i} \Delta B_{i} \quad \text { and } \quad\left(A_{i} \cap A_{j}\right) \backslash B_{j} \subset A_{j} \Delta B_{j}
$$

and

$$
A_{i} \cap A_{j} \subset\left(\left(A_{i} \cap A_{j}\right) \backslash B_{i}\right) \cup\left(\left(A_{i} \cap A_{j}\right) \backslash B_{j}\right) .
$$




\section{MCSHANE INTEGRAL AND THE RADON-NIKODYM PROPERTY}

Therefore,

$$
\sum_{i=1}^{m}\left\|F_{\mathscr{B}}\left(B_{i}\right)\right\|<\sum_{i=1}^{m}\left\|F_{\mathscr{A}}\left(A_{i}\right)\right\|+\frac{\varepsilon}{2}<M+\frac{\varepsilon}{2}+\frac{\varepsilon}{2}<M+1,
$$

where $M=\operatorname{var}_{F_{\mathscr{A}}}([0,1])$. This means that $F_{\mathscr{B}}$ is of bounded variation.

Claim 3. The vector measure $F_{\mathscr{B}}$ has an extension to a countable additive $\lambda$-continuous vector measure $F_{\mathcal{L}}: \mathcal{L} \rightarrow X$. Moreover, $F_{\mathcal{L}}: \mathcal{L} \rightarrow X$ is of bounded variation. First, we note that

$$
\mathcal{L}=\left\{B \cup Z^{\prime}: B \in \mathscr{B} \text { and } Z^{\prime} \subset Z \text { for some } Z \in \mathscr{Z}\right\},
$$

where $\mathscr{Z}=\{Z \in \mathscr{B}: \lambda(Z)=0\}$. Then, let us define $F_{\mathcal{L}}: \mathcal{L} \rightarrow X$ as follows

$$
F_{\mathcal{L}}\left(B \cup Z^{\prime}\right)=F_{\mathscr{B}}(B) \quad \text { for all } \quad B \cup Z^{\prime} \in \mathcal{L} .
$$

This is well defined, since if $B_{1} \cup Z_{1}^{\prime}=B_{2} \cup Z_{2}^{\prime}$, then $\lambda\left(B_{1} \backslash B_{2}\right)=\lambda\left(B_{2} \backslash B_{1}\right)=0$ and from this it follows that

$$
\begin{aligned}
F_{\mathscr{B}}\left(B_{1}\right) & =F_{\mathscr{B}}\left(B_{1} \backslash B_{2}\right)+F_{\mathscr{B}}\left(B_{1} \cap B_{2}\right) \\
& =F_{\mathscr{B}}\left(B_{1} \cap B_{2}\right) \\
& =F_{\mathscr{B}}\left(B_{2} \backslash B_{1}\right)+F_{\mathscr{B}}\left(B_{1} \cap B_{2}\right) \\
& =F_{\mathscr{B}}\left(B_{2}\right) .
\end{aligned}
$$

Clearly, $F_{\mathcal{L}}$ is a countable additive $\lambda$-continuous vector measure of bounded variation such that $F_{\mathcal{L}}(B)=F_{\mathscr{B}}(B)$ for all $B \in \mathscr{B}$.

Claim 4. The vector measure $F_{\mathcal{L}}$ is unique. Suppose that $F_{\mathscr{B}}$ has another extension to a countable additive $\lambda$-continuous vector measure $G_{\mathcal{L}}: \mathcal{L} \rightarrow X$. Let $B \cup Z^{\prime}$ be an arbitrary element of $\mathcal{L}$. We can assume that $B \cap Z^{\prime}=\emptyset$ (otherwise, replace $Z^{\prime}$ with $Z^{\prime} \backslash B$ ). Since the vector measure $G_{\mathcal{L}}$ is $\lambda$-continuous and $\lambda\left(Z^{\prime}\right)=0$, we obtain

$$
G_{\mathcal{L}}\left(B \cup Z^{\prime}\right)=G_{\mathcal{L}}(B)+G_{\mathcal{L}}\left(Z^{\prime}\right)=G_{\mathcal{L}}(B)=F_{\mathscr{B}}(B)=F_{\mathcal{L}}\left(B \cup Z^{\prime}\right) .
$$

Hence, we infer that $F_{\mathcal{L}}$ is unique.

Claim 5. The vector measure $F_{\mathcal{L}}$ satisfies (2.1). Indeed, since $\mathcal{I} \subset \mathscr{B}$, we obtain by (2.2) that

$$
F_{\mathscr{B}}(I)=\widetilde{F}(I) \quad \text { for all } \quad I \in \mathcal{I},
$$

and since $F_{\mathcal{L}}$ is an extension of $F_{\mathscr{B}}$ to $\mathcal{L}$, it follows that $F_{\mathcal{L}}$ satisfies (2.1) and the proof is finished. 
LEMMA 2.2. Let $X$ be a complete locally convex space and let $F:[0.1] \rightarrow X$ be a function. If $F$ is $s A C$, then there exists a unique countable additive $\lambda$-continuous vector measure $F_{\mathcal{L}}: \mathcal{L} \rightarrow X$ such that

$$
\widetilde{F}(I)=F_{\mathcal{L}}(I) \quad \text { for every } \quad I \in \mathcal{I} .
$$

Moreover, $F_{\mathcal{L}}$ is of bounded variation.

P r o o f. Since each $\phi_{p} \circ F$ is $s A C$, we obtain by Lemma 2.1 that for each $p \in \mathscr{P}$ there is a unique countable additive $\lambda$-continuous vector measure $F_{\mathcal{L}}^{(p)}: \mathcal{L} \rightarrow \bar{X}_{p}$ of bounded variation such that

$$
\left(\phi_{p} \circ \widetilde{F}\right)(I)=F_{\mathcal{L}}^{(p)}(I) \quad \text { for each } \quad I \in \mathcal{I} .
$$

Suppose that two arbitrary continuous seminorms $p$ and $q$ such that $p \leq q$ are given. Since $\bar{g}_{p q}$ is a continuous and linear map, we get that $\bar{g}_{p q} \circ F_{\mathcal{L}}^{(\bar{q})}$ is a countably additive $\lambda$-continuous vector measure of bounded variation such that

$$
\left(\phi_{p} \circ \widetilde{F}\right)(I)=\left(\bar{g}_{p q} \circ F_{\mathcal{L}}^{(q)}\right)(I) \quad \text { for each } \quad I \in \mathcal{I} .
$$

Consequently, we obtain by uniqueness of $F_{\mathcal{L}}^{(p)}$ that

$$
\bar{g}_{p q} \circ F_{\mathcal{L}}^{(q)}=F_{\mathcal{L}}^{(p)}
$$

According to [14, Theorem 5.4], for every $E \in \mathcal{L}$ there exists a unique vector $F_{\mathcal{L}}(E) \in X$ such that

$$
\phi_{p}\left(F_{\mathcal{L}}(E)\right)=F_{\mathcal{L}}^{(p)}(E) \quad \text { for all } \quad p \in \mathscr{P} .
$$

Hence, the function $F_{\mathcal{L}}: \mathcal{L} \rightarrow X$ is a countable additive $\lambda$-continuous vector measure of bounded variation, because each $F_{\mathcal{L}}^{(p)}$ is such a vector measure. By (2.4) and (2.5), for each $I \in \mathcal{I}$, we have also that

$$
\phi_{p}\left(F_{\mathcal{L}}(I)\right)=\phi_{p}(\widetilde{F}(I)) \quad \text { for all } \quad p \in \mathscr{P} .
$$

Since $X$ is Hausdorff, the last result yields

$$
F_{\mathcal{L}}(I)=\widetilde{F}(I) \quad \text { for all } \quad I \in \mathcal{I} .
$$

Thus, $F_{\mathcal{L}}$ is the desired vector measure and the proof is finished.

Now, we are ready to present the main result.

TheORem 2.3. Let $X$ be a complete locally convex space and let $F:[0,1] \rightarrow X$ be a function. Then, the following statements are equivalent:

(i) $X$ has the RNP, 


\section{MCSHANE INTEGRAL AND THE RADON-NIKODYM PROPERTY}

(ii) if we have

$$
F \in \mathcal{V}_{(p)}^{\mathcal{M}} \quad \text { for all } \quad p \in \mathscr{P},
$$

then $F$ is differentiable by seminorms,

(iii) if we have

$$
F \in \mathcal{V}_{(p)}^{\mathcal{M}} \quad \text { for all } \quad p \in \mathscr{P}
$$

then there is a variational McShane integrable function $f:[0,1] \rightarrow X$ such that

$$
\widetilde{F}(I)=(M) \int_{I} f \quad \text { for all } \quad I \in \mathcal{I},
$$

(iv) if we have

$$
F \in \mathcal{V}_{(p)}^{\mathcal{M}} \quad \text { for all } \quad p \in \mathscr{P}
$$

then there is a McShane integrable function $f:[0,1] \rightarrow X$ such that

$$
\widetilde{F}(I)=(M) \int_{I} f \quad \text { for all } \quad I \in \mathcal{I},
$$

and $f$ is weakly equivalent to a measurable by seminorm function $g$.

Proof. (i) $\Rightarrow$ (ii) Assume that for each $p \in \mathscr{P}$, we have $F \in \mathcal{V}_{(p)}^{\mathcal{M}}$. Since

$$
V_{(p, F)}^{\mathcal{M}}=V_{\phi_{p} \circ F}^{\mathcal{M}} \quad \text { for all } \quad p \in \mathscr{P},
$$

we obtain by [5, Lemma 3.1] that each $\phi_{p} \circ F$ is $s A C$ and therefore the function $F$ is $s A C$. Hence, by Lemma 2.2 , there exists a countable additive $\lambda$-continuous vector measure $\nu: \mathcal{L} \rightarrow X$ of bounded variation such that

$$
\widetilde{F}(I)=\nu(I) \quad \text { for every } \quad I \in \mathcal{I},
$$

and since $X$ has the RNP, there exists an integrable by seminorms function $f$ such that

$$
\nu(E)=\int_{E} f d \lambda \quad \text { for every } \quad E \in \mathcal{L} .
$$

Fix an arbitrary $p \in \mathscr{P}$. Then, the function $\phi_{p} \circ f$ is Bochner integrable and

$$
\left(\phi_{p} \circ \nu\right)(E)=(B) \int_{E}\left(\phi_{p} \circ f\right) d \lambda \quad \text { for all } E \in \mathcal{L},
$$

and consequently, we obtain by (2.6) that

$$
\phi_{p}(\widetilde{F}(I))=(B) \int_{I}\left(\phi_{p} \circ f\right) d \lambda \quad \text { for all } \quad I \in \mathcal{I} .
$$


Hence, by [3, Theorem II.2.9], there exists $Z_{p} \subset[0,1]$ with $\lambda\left(Z_{p}\right)=0$ such that $\left(\phi_{p} \circ F\right)^{\prime}(t)=\left(\phi_{p} \circ f\right)(t)$ for all $t \in[0,1] \backslash Z_{p}$. Since $p$ is arbitrary, it follows that $F$ is differentiable by seminorms.

(ii) $\Rightarrow$ (iii) Assume that for each $p \in \mathscr{P}$, we have $F \in \mathcal{V}_{(p)}^{\mathcal{M}}$. Then, by (ii), there exists a function $f:[0,1] \rightarrow X$ satisfying the following property: for each $p \in \mathscr{P}$, there exists a subset $Z_{p} \subset[0,1]$ with $\lambda\left(Z_{p}\right)=0$ such that

$$
\lim _{h \rightarrow 0} p\left(\frac{F(t+h)-F(t)}{h}-f(t)\right)=0 \quad \text { for all } \quad t \in[0,1] \backslash Z_{p} .
$$

Hence,

$$
\left(\phi_{p} \circ F\right)^{\prime}(t)=\left(\phi_{p} \circ f\right)(t) \quad \text { for all } t \in[0,1] \backslash Z_{p},
$$

and since $V_{\phi_{p} \circ F}^{\mathcal{M}}=V_{(p, F)}^{\mathcal{M}}$, we have also in [5, Lemma 3.1] that each $\phi_{p} \circ F$ is $s A C$. Therefore, by [13, Theorem 7.4.13], each function $\phi_{p} \circ f$ is variational McShane integrable (strongly McShane integrable) on $[0,1]$ and

$$
\left(\phi_{p} \circ \widetilde{F}\right)(I)=(M) \int_{I} \phi_{p} \circ f \quad \text { for every } \quad I \in \mathcal{I} .
$$

Therefore, we obtain by Definition 1.2 that $f$ is variational McShane integrable on $[0,1]$ and

$$
\widetilde{F}(I)=(M) \int_{I} f \quad \text { for every } \quad I \in \mathcal{I} .
$$

(iii) $\Rightarrow$ (iv) Assume that for each $p \in \mathscr{P}$, we have $F \in \mathcal{V}_{(p)}^{\mathcal{M}}$. Then, we obtain by (iii) that there exists a variational McShane integrable function $f:[0,1] \rightarrow X$ such that $\widetilde{F}(I)=(M) \int_{I} f$, for all $I \in \mathcal{I}$. Then, by Definition 1.2, each function $\phi_{p} \circ f$ is variational McShane integrable and therefore, we obtain by [13, Theorem 5.1.4] that each function $\phi_{p} \circ f$ is Bochner integrable. Fix an arbitrary $p \in \mathscr{P}$. Since $\phi_{p} \circ f$ is measurable, we can choose a sequence $\left(\widetilde{f}_{k}^{(p)}\right)_{k}$ of measurable simple functions which converges pointwise almost everywhere to $\phi_{p} \circ f$ and

$$
\widetilde{f}_{k}^{(p)}([0,1]) \subset\left(\phi_{p} \circ f\right)([0,1]) \quad \text { for all } \quad k \in \mathbb{N} .
$$

Therefore, we can define a sequence $\left(f_{k}^{(p)}\right)_{k}$ of measurable simple functions such that $\tilde{f}_{k}^{(p)}=\phi_{p} \circ f_{k}^{(p)}$, for all $k \in \mathbb{N}$. Hence,

$$
\lim _{k \rightarrow \infty} p\left(f_{k}^{(p)}(t)-f(t)\right)=0 \quad \lambda \text { - a.e. on }[0,1] \text {. }
$$

Since $p$ is arbitrary, the last result yields that $f$ is measurable by seminorms. Thus, $f$ is weakly equivalent to the measurable by seminorms function $f$. 


\section{MCSHANE INTEGRAL AND THE RADON-NIKODYM PROPERTY}

(iv) $\Rightarrow$ (i) Let $\nu: \mathcal{L} \rightarrow V$ be a countable additive $\lambda$-continuous vector measure of bounded variation. We define a function $F:[0,1] \rightarrow X$ as follows

$$
\widetilde{F}(I)=\nu(I) \quad \text { for all } \quad I \in \mathcal{I} \text {. }
$$

Fix an arbitrary $p \in \mathscr{P}$. Since $\phi_{p} \circ \nu$ is an countable additive $\lambda$-continuous vector measure of bounded variation, its variation $\left|\phi_{p} \circ \nu\right|$ is a $\lambda$-continuous finite measure. Therefore, we obtain by [12, Theorem 6.11] that to a given $\varepsilon>0$, there exists $\eta_{p}>0$ such that for every $E \in \mathcal{L}$, we have

$$
\lambda(E)<\eta_{p} \Rightarrow\left|\phi_{p} \circ \nu\right|(E)<\varepsilon .
$$

Let $D$ be a finite collection of pairwise nonoverlapping intervals in $\mathcal{I}$ such that $\sum_{I \in D} \lambda(I)<\eta_{p}$. Then,

$$
\sum_{I \in D} \widetilde{p}\left(\left(\phi_{p} \circ \widetilde{F}\right)(I)\right) \leq \sum_{I \in D}\left|\phi_{p} \circ \nu\right|(I)=\left|\phi_{p} \circ \nu\right|\left(\bigcup_{I \in D} I\right)<\varepsilon .
$$

This means that $\phi_{p} \circ \widetilde{F}$ is $s A C$. Hence, we obtain by [5, Lemma 3.1] that

$$
F \in V_{p}^{\mathcal{M}},
$$

and since $p$ is arbitrary, the last result together with $(i v)$ yields that there exists a McShane integrable function $f:[0,1] \rightarrow X$ such that

$$
\widetilde{F}(I)=(M) \int_{I} f \quad \text { for every } \quad I \in \mathcal{I}
$$

and $f$ is weakly equivalent to a measurable by seminorms function $g:[0,1] \rightarrow X$.

Since $f:[0,1] \rightarrow X$ is McShane integrable, we obtain by Definition 1.1 that each function $\phi_{p} \circ f$ is McShane integrable and

$$
\left(\phi_{p} \circ \widetilde{F}\right)(I)=(M) \int_{I} \phi_{p} \circ f \quad \text { for every } \quad I \in \mathcal{I} .
$$

By (1.1), we have also that $\phi_{p} \circ f$ is weakly equivalent to $\phi_{p} \circ g$. Now, by the same manner as in Theorem $3.1((\mathrm{iv}) \Rightarrow(\mathrm{i}))$ in [5], we get that the function $\phi_{p} \circ g$ is Bochner integrable and

$$
\left(\phi_{p} \circ \nu\right)(E)=(B) \int_{E}\left(\phi_{p} \circ g\right) d \lambda \quad \text { for every } E \in \mathcal{L} .
$$

We will show that $g$ is integrable by seminorms. Since $\phi_{p} \circ g$ is measurable, we can define a sequence $\left(g_{k}^{(p)}\right)$ of measurable simple functions such that

$$
\left(\phi_{p} \circ g_{k}^{(p)}\right)([0,1]) \subset\left(\phi_{p} \circ g\right)([0,1]) \quad \text { for all } \quad k \in \mathbb{N}
$$


and the equalities

$$
\lim _{k \rightarrow \infty} p\left(g_{k}^{(p)}(t)-g(t)\right)=\lim _{k \rightarrow \infty} \tilde{p}\left(\left(\phi_{p} \circ g_{k}^{(p)}\right)(t)-\left(\phi_{p} \circ g\right)(t)\right)=0
$$

hold true at almost all $t \in[0,1]$. We have also that $\phi_{p} \circ g$ is almost everywhere bounded on $[0,1]$. Therefore, we obtain by Dominated Convergence Theorem, [3, Theorem II.2.3], that

$$
\lim _{k \rightarrow \infty} \tilde{p}\left(\int_{E}\left(\phi_{p} \circ g_{k}^{(p)}\right) d \lambda-(B) \int_{E}\left(\phi_{p} \circ g\right) d \lambda\right)=0
$$

and

$$
\lim _{k \rightarrow \infty} \int p\left(g_{k}^{(p)}(t)-f(t)\right) d \lambda=0 .
$$

By (2.8) and (2.10), we get

$$
\lim _{k \rightarrow \infty} p\left(\int_{E} g_{k}^{(p)} d \lambda-\nu(E)\right)=0
$$

By Definition 1.3, since $p$ is arbitrary, the last equality together with (2.9) and (2.11) yields that $g$ is integrable by seminorms and

$$
\nu(E)=\int_{E} g d \lambda \quad \text { for each } \quad E \in \mathcal{L} .
$$

This proves that $X$ has the RNP and the proof is finished.

\section{REFERENCES}

[1] BLONDIA, C.: Integration in locally convex spaces, Simon Stevin 55 (1981), 81-102.

[2] BONGIORNO, B.-DI PIAZZA, L.-MUSIAL, K.: A variational Henstock integral characterization of the Radon-Nikodym property, Illinois J. Math. 53 (2009), 87-99.

[3] DiEsteL, J.-UHL, J. J.: Vector Measures, in: Math. Surveys Monogr., Vol. 15, Amer. Math. Soc., Providence, RI, 1977.

[4] DUNFORD, N.-SCHWARTZ, J. T.: Liner Operators, Part I: General Theory, in: Pure Appl. Math. (N.Y.), Vol. 7, Interscience Publ., New York, 1958.

[5] KALIAJ, S. B.: A variational McShane integral characterization of the Radon-Nikodym property, Math. Slovaca 63 (2013), 503-510.

[6] KALIAJ, S. B.: The Radon-Nikodym property and the limit average range, Math. Slovaca (to appear).

[7] KALIAJ, S. B.: The average range characterization of the Radon-Nikodym property, Mediterr. J. Math., Springer, Basel, 2013, DOI 10.1007/ s00009-013-0352-3.

[8] KALIAJ, S. B.: The Radon-Nikodym property, generalized bounded variation and the average range, Math. Nachr. (2014), 1-9, /DOI 10.1002/mana.201300171. 


\section{MCSHANE INTEGRAL AND THE RADON-NIKODYM PROPERTY}

[9] MARRAFFA, V.: Riemann type integrals for functions taking values in a locally convex space, Czechoslovak Math. J. 56 (2006), 475-490.

[10] MARRAFFA, V.: Non absolutely convergent integrals of functions taking values in a locally convex space, Rocky Mountain J. Math. 36 (2006), 1577-1593.

[11] MARRAFFA, V.: The variational McShane integral, Rocky Mountain J. Math. 39 (2009), 1993-2013.

[12] RUDIN, W.: Real and Complex Analysis (2nd ed.), McGraw-Hill Book Comp., New York, 1974.

[13] SCHWABIK, Š.-YE, G.: Topics in Banach Space Integration, in: Ser. Real Anal., Vol. 10, World Scientific, Hackensack, NJ, 2005.

[14] SCHAEFER, H. H.: Topological Vector Spaces, Grad. Texts in Math., Vol. 3 (3rd printing corrected), Springer-Verlag XI, New York, (1971).

[15] THOMSON, B. S.: Derivates of Interval Functions, Mem. Amer. Math. Soc. (1991), 452.

[16] THOMSON, B. S.: Differentiation, pp. 179-247 in: Handb. Measure Theory Vol. I and II. (E. Pap, ed.), North-Holland, Amsterdam, 2002, pp. 179-247.

Received May 1, 2013

University of Elbasan

Science Natural Faculty

Mathematics Department

Elbasan

ALBANIA

E-mail: sokol_bush@yahoo.co.uk 